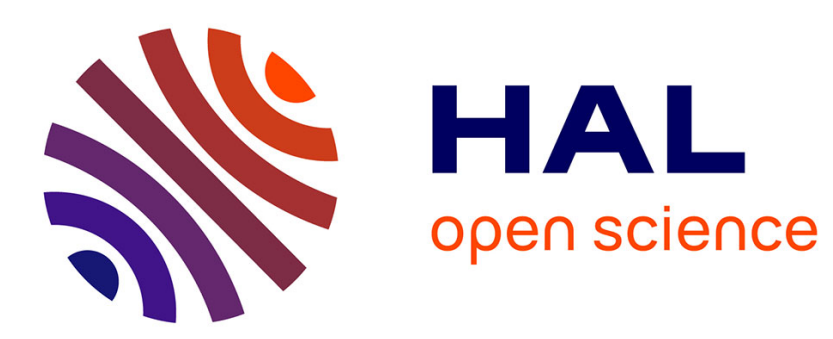

\title{
Efficacité d'un feedback hebdomadaire sur la réduction du poids des ordures ménagères résiduelles
}

Sébastien Meineri, I. Dangeard, M. Dupré

\section{To cite this version:}

Sébastien Meineri, I. Dangeard, M. Dupré. Efficacité d'un feedback hebdomadaire sur la réduction du poids des ordures ménagères résiduelles. Pratiques Psychologiques, 2018, 24 (1), pp.79-97. 10.1016/j.prps.2016.11.004 . hal-01960243

\section{HAL Id: hal-01960243 \\ https://hal.univ-rennes2.fr/hal-01960243}

Submitted on 19 Dec 2018

HAL is a multi-disciplinary open access archive for the deposit and dissemination of scientific research documents, whether they are published or not. The documents may come from teaching and research institutions in France or abroad, or from public or private research centers.
L'archive ouverte pluridisciplinaire HAL, est destinée au dépôt et à la diffusion de documents scientifiques de niveau recherche, publiés ou non, émanant des établissements d'enseignement et de recherche français ou étrangers, des laboratoires publics ou privés. 


\title{
Efficacité d'un feedback hebdomadaire sur la réduction du poids des ordures ménagères résiduelles
}

Effectiveness of a weekly feedback on household residual waste reduction

\author{
S. Meineri ${ }^{\mathrm{a}, *}$, I. Dangeard $^{\mathrm{b}}$, M. Dupré ${ }^{\mathrm{a}}$ \\ a Laboratoire CRPCC, université Bretagne-Sud, campus de Tohannic, rue André-Lwoff, \\ BP 573, 56017 Vannes cedex, France \\ ${ }^{\mathrm{b}}$ Laboratoire EREID, université de Bretagne Occidentale, 29200 Brest, France
}

Reçu le 30 mai 2016 ; accepté le 22 novembre 2016

\section{Résumé}

Introduction. - Jusqu'alors centrée sur la seule pratique du tri sélectif, la gestion des déchets intègre depuis peu ses composantes complémentaires : réutilisation des biens et réduction à la source.

Objectif. - Dans le contexte législatif du développement de la redevance incitative sur le poids des Ordures ménagères résiduelles (OMR), notre recherche visait à évaluer la pertinence d'une approche alternative moins coercitive basée sur un feedback personnalisé.

Méthode. - Trente-quatre foyers, repartis en deux conditions ont pris part à l'expérimentation. Les foyers de la condition feedback ont reçu durant 14 semaines une information hebdomadaire sur le poids effectif de leurs OMR tandis que ceux de la condition contrôle n'ont reçu aucune information.

Résultats. - Les foyers de la condition feedback ont réduit le poids de leurs OMR entre la période préexpérimentale et la période expérimentale significativement plus que ceux de la condition contrôle.

Discussion. - L'interview des foyers et l'inventaire des recherches menées sur le feedback permettent de proposer des pistes d'améliorations et des recherches futures.

C 2016 Société Française de Psychologie. Publié par Elsevier Masson SAS. Tous droits réservés.

Mots clés : Gestion des déchets ; Réduction des déchets ; Feedback ; Redevance incitative

\section{Abstract}

Introduction. - Historically focused on waste sorting, waste management recently integrates its complementary components: reuse and reduction.

\footnotetext{
* Auteur correspondant.

Adresse e-mail : sebastien.meineri@univ-ubs.fr (S. Meineri).
} 
Objective. - "Pay as you throw" systems are presently fostered by the French prevailing legislation. In this context, our research was designed to assess the relevance of a less coercive approach based on tailored feedback.

Method. - Thirty-four households took part in the experiment and were divided into two conditions. Households in the feedback condition received weekly information about their residual waste weight during 14 weeks while those in the control condition received no information.

Results. - Households in the feedback condition reduced their waste weight between the baseline period and the experimental period significantly more than the control condition.

Discussion. - Interviews of participants and literature review about the feedback approach enables us to suggest improvements and relevant futures studies.

(C) 2016 Société Française de Psychologie. Published by Elsevier Masson SAS. All rights reserved.

Keywords: Waste management; Waste reduction; Feedback; Pay-as-you-throw

\section{Introduction}

Les préoccupations relatives à l'état de l'environnement se sont fortement développées depuis trois décennies. Parmi les différents enjeux d'un développement plus soutenable, celui de la réduction des déchets est apparu dès le sommet de la terre de Rio en 1992. En 2012, 345 millions de tonnes de déchets ont été produites en France, dont 31 millions de tonnes par les ménages (ADEME, 2015). En 2013, on estimait la production de déchets par an et par habitant à $530 \mathrm{~kg}$ en France (EUROSTAT, 2015). La gestion des déchets a, dans un premier temps, été considérée et étudiée sous le seul angle du tri des déchets. Depuis peu, elle est envisagée de manière plus globale, en intégrant les dimensions réutilisation et réduction à la source (EEA, 2014). La présente recherche s'inscrit dans le cadre de cette évolution récente des études et vise à tester l'effet d'une stratégie permettant de réduire le poids des Ordures ménagères résiduelles (OMR), i.e. la part de déchets ne pouvant être valorisée dans les circuits de recyclage. Le recensement des nombreuses recherches réalisées sur la dimension recyclage et les données disponibles sur les dimensions réutilisation et réduction serviront d'appui théorique et méthodologique à notre recherche.

\subsection{La gestion des déchets sous l'angle du recyclage}

La littérature scientifique est très fournie en ce qui concerne la pratique de tri, qu'il s'agisse des caractéristiques individuelles et facteurs sociodémographiques (Schultz, Oskamp, \& Mainieri, 1995) ou de l'efficacité d'interventions spécifiques (Dupré, Dangeard, \& Meineri, 2014a, 2014b).

Trois catégories d'interventions coexistent dans la littérature : les stratégies informationnelles, incitatives et comportementales. Les stratégies informationnelles se caractérisent par la diffusion d'informations sur l'état de l'environnement et les modalités d'action à disposition des individus pour faire face à ses conséquences sociétales, collectives ou individuelles, néfastes. Les recherches mobilisant le modèle du comportement planifié (Ajzen, 1991), telles que Humphrey, Bord, Hammond, et Mann (1977), Tonglet, Phillips, et Read (2004), Nigbur, Lyons, et Uzzell (2005) ou encore Botetzagias, Dima, et Malesios (2015), établissent un lien positif entre les cognitions relatives au recyclage (attitudes, perceptions des normes et du contrôle), et l'intention de tri. De fait, elles laissent envisager qu'en modifiant les croyances des individus, on parviendrait à 
modifier leurs comportements. Malgré certains succès (Jacobs \& Bailey, 1982 ; Luyben \& Bailey, 1979 ; Burn \& Oskamp, 2013 ; Hopper \& Nielsen, 1991 ; Jacobs, Bailey, \& Crews, 1984), les auteurs s'accordent à penser que la seule diffusion d'informations peut dans une certaine mesure favoriser la pérennité d'un comportement déjà en place (Geller, 1992) mais ne suffit pas à produire un changement conséquent (Nickerson, 2003 ; Gardner \& Stern, 1996 ; Bamberg \& Moser, 2007). Le lien indirect et modéré entre les cognitions et les comportements effectifs expliquerait l'effet limité de ces stratégies (Webb \& Sheeran, 2006 ; Davies, Foxall, \& Pallister, 2002).

Les stratégies incitatives se caractérisent par l'attribution d'une récompense aux individus réalisant le comportement attendu. Fondée sur les théories béhavioristes (Thorndike, 1998 ; Skinner, 1953) postulant qu'un renforcement positif favorise la répétition d'un comportement, et qu'à l'inverse un renforcement négatif inhibe son émergence, elles présentent généralement un effet positif sur les pratiques de recyclage, et ce, avec différents types de récompense : chèque cadeaux, billets de loterie, bons de réduction (Timlett \& Williams, 2008 ; Geller, Chaffee, \& Ingram, 1975 ; Hamad, Bettinger, Cooper, \& Semb, 1980 ; Witmer \& Geller, 1976). L'effet serait en outre accru dès lors que la stratégie est couplée à de l'information persuasive (Geller, 1992) et s'adresse à des populations dont le niveau de participation est initialement très faible (Woodard, Firoozmand, \& Harder, 2006). Deux limites sont toutefois à relever : d'une part, les recherches mesurant l'effet à moyen terme font montre d'une disparition une fois la récompense obtenu (Iyer \& Kashyap, 2007 ; Oskamp, 1995 ; Pardini \& Katzev, 1983 ; Katzev \& Pardini, 1988 ; De Young, 1990) ; d'autre part, l'effet obtenu sur la période d'incitation se limite au seul comportement rétribué. Needleman et Geller (1992) révèlent ainsi qu'une incitation financière associée au tri de canettes métalliques ne produit aucun effet sur le recyclage du papier ou du verre. Hong (1999) observe que l'efficacité de la récompense sur le tri des déchets nuit à la réduction des déchets à la source. Constituant une motivation extrinsèque, la récompense inhiberait la recherche de justifications internes (Deci \& Ryan, 1988), garantes de la pérennité et de la généralisation du comportement à des registres comportementaux proches.

Enfin, les stratégies comportementales, opérationnalisant la théorie de l'engagement (Kiesler, 1971 ; Joule \& Beauvois, 1998) se caractérisent par l'obtention d'un comportement dit préparatoire, réalisé dans des circonstances spécifiques (cf. facteurs d'engagement : comportement coûteux, répété, irrévocable, explicite et volontaire). Ce comportement préalable aurait pour effet de tisser un lien avec l'individu et l'engagerait dans un cours d'action, favorisant l'apparition du ou des comportements attendus. Les résultats de cette approche présentent un effet globalement positif sur les pratiques de tri (Burn \& Oskamp, 2013 ; Porter, Leeming, \& Dwyer, 1995 ; Dupré, 2009 ; Katzev \& Pardini, 1988 ; Wang \& Katzev, 1990 ; Werner et al., 1995 ; Zbinden, Souchet, Girandola, \& Bourg, 2011 ; Dupré, Meineri, \& Guéguen, 2014a, 2014b ; Guéguen, Meineri, Martin, \& Grandjean, 2010). Deux types de comportements préparatoires sont généralement utilisés : d'une part, un engagement, individuel ou collectif, oral ou écrit à participer à une opération de tri sur une période donnée ; d'autre part, un questionnaire d'une dizaine de questions administré dans le cadre d'un sondage préalable. Contrairement aux stratégies incitatives, les mesures à moyen terme révèlent une pérennité de l'effet au-delà de la période initiale d'engagement (Katzev \& Pardini, 1988 ; Wang \& Katzev, 1990). Le comportement amorcé par l'engagement ou l'acte initial trouverait par la suite des justifications internes (Pallak \& Cummings, 1976).

À l'interface des 3 approches présentées, la technique du feedback a également fait l'objet d'un certain nombre de travaux. À l'instar des stratégies informationnelles, le feedback se caractérise par la transmission d'une information. Mais celle-ci est transmise régulièrement et est relative au comportement spécifique du récepteur. À l'instar des stratégies incitatives, cette information sanctionne une performance mais à la différence de celles-ci aucune récompense matérielle n’y 
est associée. Dès lors, et sur ce dernier aspect, le feedback se rapprocherait également des stratégies comportementales. L'individu agissant dans des circonstances théoriquement engageantes (comportement coûteux, répété, et réalisé dans un contexte de liberté notamment), les premiers efforts pour augmenter sa performance l'inciteraient à accroître ceux-ci et trouver des justifications internes pour les poursuivre. Initialement testée sur la diminution des consommations d'énergie (Winett, Neale, \& Grier, 1979 ; Katzev, Cooper, \& Fisher, 1981 ; Darby, 2006), le feedback a également fait l'objet de quelques recherches sur la pratique de tri. Les résultats présentent globalement un effet positif, que le feedback soit collectif (Katzev \& Mishima, 1992 ; Hamad et al., 1980 ; Goldenhar \& Connel, 1991-1992), individuel (Fuqua \& Deleon, 1995), régulier sur plusieurs semaines (Schultz, 1999; Fuqua \& Deleon, 1995) ou ponctuel (Perrin \& Barton, 2001). Timlett \& Williams (2008) observent pour leur part un effet positif d'un feedback portant non sur la quantité de tri comme précédemment mais sur la qualité de celui-ci. L'effet ne touche cependant pas les non-trieurs initiaux. Concernant les effets à long terme d'un feedback quantitatif, Schultz (1999) mesurant les données à 4 semaines après la période expérimentale initiale de 12 semaines, observe un maintien global de l'augmentation du poids de déchets triés malgré un effet rebond auprès des foyers les plus performants initialement.

\subsection{La gestion des déchets sous l'angle de la réutilisation et de la réduction à la source}

Si le développement du recyclage a permis de détourner $28 \%$ des déchets nord-américains de l'enfouissement, $72 \%$ de ceux-ci, dits Ordures ménagères résiduelles (OMR), restent encore à traiter (Lehman \& Geller, 2005). En France, en 2013, $26 \%$ des déchets étaient pris en charge par les filières de recyclage et valorisés sous forme de matière (ADEME, 2015). Bien que relevant du même objet, la réutilisation des biens et la réduction des déchets à la source permettant de réduire la part d'OMR n'ont pas fait l'objet de la même attention que le recyclage (Nickerson, 2003). Effectivement distinguées par Barr, Gilg, \& Ford (2001), les trois dimensions de la problématique de gestion des déchets ne présentent pas une forte consistance qu'il s'agisse des intentions ou des comportements déclarés. Également, les facteurs explicatifs du tri, de la réutilisation des biens ou de la réduction tendent à différer (Barr et al., 2001 ; Tonglet et al., 2004). Barr et al. (2001) indiquent que la réduction des déchets, pratique moins fréquente et plus marginale que le tri, nécessiterait un niveau de préoccupation environnementale particulièrement élevé. Robertson et Walkington (2009) pointent pour leur part, outre la nécessité d'une information, une influence déterminante des motivations intrinsèques.

Concernant les stratégies mises en ouvre pour stimuler la réduction des OMR, outre l'information et la sensibilisation, un certain nombre de collectivités à l'international ont adopté des systèmes de tarification incitative consistant à intégrer dans le calcul des charges imputées au ménages, une part variable basée sur les déchets effectivement produits par le foyer. Selon les cas, cette part variable peut être calculée à l'aide du nombre de présentation des conteneurs, du volume des conteneurs, du poids de déchets enregistrés par des dispositifs de pesée embarqués à bord des véhicules de collecte, de la distribution de sacs spécifiques, ou encore d'étiquettes à apposer sur les sacs. L'effet de la mise en œuvre de cet outil lorsqu'il est évalué, se révèle plutôt positif avec une diminution du poids de déchets de 20 à $30 \%$ (Callan \& Thomas, 2006 ; Dahlén, Åberg, Lagerkvist, \& Berg, 2009 ; Allers et Hoeben, 2010) accompagné d'une augmentation des déchets triés. Cependant, certaines recherches pointent également les limites en repérant des dépôts sauvages ou des dépôts sur les communes adjacentes plus ou moins marqués (Fullerton \& Kinnaman, 1996 ; Linderhof, Kooreman, Allers, \& Wiersma, 2001). En France, le développement de la tarification incitative, possible sur le plan législatif depuis 1976, est relativement 
récente. Elle fait suite aux impulsions données par les lois Grenelle I (2009), Grenelle II (2010), la LTECV (loi relative à la transition énergétique pour la croissance verte) (2015) et surtout au déblocage d'aides financières par l'ADEME (Gatier, 2016). En 2014, 4,2 millions d'habitants dans 159 collectivités françaises étaient ainsi soumis à une redevance incitative, dont la part variable était majoritairement basée sur le nombre de présentations de conteneurs. Le résultat de la mise en place de cet outil est globalement positif (enquête « collecte » ADEME présentée dans Gatier, 2016), conformément aux études internationales citées. Plus précisément, l'effet positif s'observe dès que la collectivité commence à communiquer sur l'outil, se poursuit avec sa mise en place effective de celui-ci jusqu'à atteindre un seuil. La diminution de la quantité d'OMR s'accompagne en outre d'une augmentation des déchets recyclés. Toutefois, à l'instar des limites pointées dans les recherches citées, les données présentent une certaine dégradation de la qualité des déchets triés, particulièrement marquée sur les deux premières années, sans que l'on puisse statuer sur le caractère volontaire ou non des erreurs. Du point de vue des collectivités, la mise en œuvre de la tarification incitative peut s'avérer relativement longue, coûteuse et complexe selon le choix du mode de calcul de la part variable (nombre de présentations des conteneurs, poids des déchets...) et le système de tarification initialement en place (taxe sur l'enlèvement des ordures ménagères [TEOM], prenant comme assiette la valeur locative du logement, les recettes de la taxe pouvant être inférieures au coût du service ; ou redevance sur l'enlèvement des ordures ménagères $[\mathrm{REOM}]$ prenant généralement comme assiette le nombre d'occupants du logement et imposant un équilibre du budget déchet). En effet, l'intégration d'une part incitative, possible pour la REOM depuis 1976, ne l'est pour la TEOM que depuis 2012 ; en outre limité à $45 \%$ du recouvrement total. Sachant qu'en 2011, près de $90 \%$ des collectivités fonctionnaient sur la base du système TEOM, les difficultés inhérentes au changement de système ont conduit certaines collectivités à finalement abandonner la mise en place de l'outil (Gatier, 2016).

\subsection{Problématique et objectifs de l'étude}

L'engouement actuel des instances environnementales pour la redevance incitative et la connaissance des limites à la fois théoriques (cf. stratégie incitatives) et pratiques de cette approche nous ont conduit à nous questionner sur l'efficacité d'une approche alternative relativement similaire. De fait, la stratégie du feedback se base sur le recueil de la même information que celle utilisée pour la redevance incitative. Pourtant, elle n'a, à notre connaissance, fait l'objet d'aucune recherche en ce qui concerne le poids des déchets résiduels. La présente recherche visait principalement à tester l'efficacité d'un feedback relatif au poids des ordures ménagères résiduelles sur leur réduction dans l'agglomération de Rennes Métropole, de sorte à évaluer la pertinence de cette approche, alternative à la redevance incitative, permettant d'atteindre un même niveau d'efficacité tout en inhibant les contraintes de cette dernière. L'agglomération Rennes Métropole dispose en effet d'un système de pesée intégré aux véhicules de collecte des ordures ménagères, qui lors de chaque prise de conteneur, scanne une puce électronique située sur celui-ci et renseigne une base de données sur le poids des déchets. Les données collectées par ce système, initialement installé en prévision de l'instauration de la redevance incitative, ne sont pour l'heure pas utilisées, d'une part par crainte d'être confronté aux dérives pointées, et d'autre part, par choix politique. À la lumière des recherches réalisées sur les consommations d'énergie et sur le recyclage des déchets, nous faisions l'hypothèse d'un effet positif du feedback. Un objectif secondaire était également poursuivi dans cette recherche visant à enrichir la littérature sur la réduction des déchets en investiguant 
les liens existants entre des indicateurs attitudinaux, comportementaux de type auto-rapportées et des indicateurs réels de la gestion des déchets.

\section{Méthodologie}

\subsection{Recrutement et participants}

La population a été échantillonnée à partir des fichiers de pesée fournis par l'agglomération Rennes Métropole. Parmi les foyers habitant en maison individuelle, ceux présentant un nombre de pesées hebdomadaire supérieurs à 20 sur l'année 2013 ont été retenus. Parmi ceux-ci, 130 ont été abordés en porte à porte en dehors des horaires professionnels par une expérimentatrice se présentant comme stagiaire à l'université de Bretagne. L'expérimentatrice disait mener une étude en partenariat avec l'agglomération Rennes Métropole sur l'environnement et la gestion des déchets, et soumettait aux foyers deux requêtes consécutives sous la forme d'une procédure de Pied-dansla-porte (Freedman \& Fraser, 1966) ${ }^{1}$. La première requête consistait en un questionnaire relatif aux attitudes et comportements pro-environnementaux. La seconde requête, formulée suite à l'acceptation et au remplissage du questionnaire concernait la participation à l'étude proprement dite. Celle-ci était présentée de manière sommaire tel le test d'un nouvel outil d'aide à la gestion des déchets, pour lequel les foyers auraient à livrer ultérieurement leur ressenti. Les foyers volontaires devaient alors remplir et signer une fiche de participation en indiquant leur courriel, numéros de téléphone fixe et portable, âge, ainsi que le nombre de personnes résidant au foyer.

Cinquante-trois pour cent (69/130) des personnes sollicitées ont accepté de répondre au questionnaire. Le répondant «type » est une femme (66,7 \%), âgée de 38,1 ans (ET 10,31 ans), mariée (60,9\%), appartenant à la catégorie socioprofessionnelle des employés $(53,6 \%)$ et issue d'un foyer d'environ 4 personnes $(M=3,59$; ET =1,07). Un pourcentage de 55,1 (38/69) des répondants au questionnaire ont accepté de participer au projet. Le participant «type » est globalement le même que le répondant «type » au questionnaire : une femme (60,5\%), âgée de 37,4 ans (ET 10,31 ans), mariée (60,9\%), appartenant à la catégorie socioprofessionnelle des employés $(44,7 \%)$ et issue d'un foyer d'environ 4 personnes $(\mathrm{M}=3,66$; ET $=1,19)$.

\subsection{Procédure et variables considérées}

Cinq semaines après la fin de la phase de recrutement, les foyers volontaires recevaient un courrier les informant de la prise en considération de leur candidature.

Deux conditions expérimentales étaient définies en référence à une variable indépendante «Feedback » comprenant deux modalités : «Présence » versus « Absence ». Les foyers étaient répartis de façon aléatoire entre les deux conditions. En condition contrôle, il était dit aux foyers que l'étude avait pris du retard et qu'ils seraient recontactés ultérieurement tandis qu'en condition expérimentale, il était dit aux foyers que l'étude débutait dans les jours à venir et l'outil leur était présenté, en précisant qu'ils recevraient chaque semaine, et ce, durant les 14 semaines à venir, par courriel ainsi que par SMS le poids de déchets relevés lors de chaque collecte. De plus, il leur était expliqué que le courriel présenterait un historique actualisé des relevés semaine après semaine.

\footnotetext{
${ }^{1}$ L'opérationnalisation du recrutement sous la forme d'un PDLP visait à favoriser l'acceptation de la requête de participation à l'étude et limiter ainsi un éventuel biais d'autosélection.
} 
Cinq à sept semaines après la fin de la période de feedback, les foyers de la condition expérimentale étaient contactés par téléphone pour un entretien semi-directif permettant de recueillir leurs perceptions liées à cette expérience.

La variable dépendante de l'expérimentation était constituée par le poids des déchets résiduels relevé lors des collectes dans les deux conditions selon 4 périodes de mesure :

- période pré-expérimentale : 7 semaines précédant la période de feedback ;

- période expérimentale : 14 semaines de feedback ;

- période post-expérimentale 1 : 14 semaines suivant la période de feedback ;

- période post-expérimentale $2: 14$ semaines suivant la période post-expérimentale 1 .

\subsection{Matériel}

Le questionnaire proposé lors du recrutement comprenait 2 échelles :

- la première échelle (Annexe 1) consistait en une traduction par nos soins de l'échelle New Environmental Paradigm (NEP) dans sa version courte (Dunlap, Liere, Mertig, \& Jones, 2000). Elle mesurait l'attitude des individus à l'égard de l'environnement à l'aide de 6 affirmations (3 négatives et 3 positives) pour lesquelles les individus devaient donner leur degré d'accord sur des échelles de likert en 5 points de 1 («Pas du tout d'accord avec l'affirmation ») à 5 («Tout à fait d'accord avec l'affirmation»);

- la seconde échelle (Annexe 2) était traduite et adaptée de l'échelle 3R (Reduce, Reuse, Recycle) de Barr et al. (2001) mesurant la fréquence de réalisation de comportements relatifs à la gestion des déchets. Deux items relatifs au compostage des déchets et des restes alimentaires ont été ajoutés, tandis que l'item relatif au recyclage des boîtes de conserve a été agrégé à celui relatif au recyclage des canettes. L'échelle finale mesurait la fréquence de réalisation de 20 comportements à l'aide d'échelle de likert en 5 points de 1 (« Jamais ») à 5 (« Toujours »).

La grille d'entretien administrée suite à la période de feedback visait à cerner le ressenti des participants concernant l'outil et son utilisation, l'évolution perçue de leurs comportements et à faire émerger leurs suggestions et commentaires. La trame d'entretien posait les questions suivantes :

- avez-vous bien reçu les mails et SMS que nous vous avons envoyés ?

- avez-vous l'impression que le fait de recevoir ces informations a changé quelque chose pour vous?

○ si oui, quoi ?

- avez-vous des suggestions, ou des commentaires ?

$\circ$ fréquence et mode de réception de l'information.

\subsection{Hypothèses sur les données quantitatives}

Sur la base des données issues de la littérature relative à la procédure de feedback, nous envisagions un effet positif (H1) et durable (H2) de la procédure. Plus précisément, nous formulions les hypothèses suivantes : 
- hypothèse 1a : les foyers de la condition Feedback produiront moins de déchets que ceux de la condition Contrôle sur la période expérimentale ;

- hypothèse $1 \mathrm{~b}$ : les foyers de la condition Feedback diminueront significativement plus le poids de leur déchets entre la période pré-expérimentale et la période expérimentale que les foyers de la condition Contrôle ;

- hypothèse $2 \mathrm{a}$ : les foyers de la condition Feedback produiront moins de déchets que ceux de la condition Contrôle sur les périodes post-expérimentale 1 et 2 ;

- hypothèse $2 b$ : les foyers de la condition Feedback diminueront significativement plus le poids de leur déchets entre la période pré-expérimentale et les périodes post-expérimentales 1 et 2 que les foyers de la condition Contrôle.

Outre le test des hypothèses formulées, des analyses exploratoires seront réalisées de sorte à cerner les liens existant entre les données directes relatives au poids des déchets résiduels et celles recueillies via les échelles d'attitude (NEP) et de gestion des déchets (3R adaptée).

\section{Résultats}

\subsection{Données quantitatives}

\subsubsection{Fiabilité et dimensions des échelles du questionnaire préalable}

Les analyses de fiabilité et de catégorisation des items des échelles ont été réalisées sur l'ensemble des données disponibles, soit les 69 répondants au questionnaire.

L'échelle NEP (Dunlap et al., 2000) ne présentant qu' une seule dimension. Un unique score global agrégeant les 6 items de l'échelle a été calculé. La mesure de fiabilité d'échelle s'est révélée peu satisfaisante (Alpha de Cronbach $=.512$ ). Le retrait d'un item n'améliorant pas considérablement le niveau de fiabilité, l'ensemble des items a été conservé.

L'échelle 3R (Barr et al., 2001) adaptée a fait l'objet d'une analyse factorielle en composante principale avec rotation Varimax. Deux facteurs (au lieu de 3 dans l'échelle initiale de Barr et al., 2001) ont émergé de l'analyse expliquant $34,89 \%$ de la variance totale.

Le premier facteur que nous avons nommé « Recyclage » comprend 8 items et explique 21,34\% de la variance. L'analyse de fiabilité d'échelle présente un Alpha de Cronbach satisfaisant de : .737 (après le retrait de l'item 10).

Le second facteur, que nous avons nommé « réduction et réutilisation » comprend 12 items et explique pour sa part $13,55 \%$ de la variance totale. L'analyse de fiabilité d'échelle présente un alpha de Cronbach satisfaisant de .702 (après retrait de l'item 22) (Tableau 1).

\subsubsection{Comparabilité des participants et niveau de sensibilité environnementale a priori}

Parmi les 69 répondants du questionnaire, 38 ont accepté de participer à l'étude proprement dite $(55,1 \%)$. Les analyses réalisées n'ont pas révélé de différence statistique sur les échelles considérées selon l'acceptation ou le refus de participer au projet (échelle NEP : $[\mathrm{t}(1,68)=.348$; $p=.729 \mathrm{NS}]$; échelle 3R adaptée dimension Recyclage : $[\mathrm{t}(1,68)=1,106 ; p=.27 \mathrm{NS}]$; dimension « réutilisation et réduction »: $[\mathrm{t}(1,68)=.214 ; p=.83 \mathrm{NS}])$. Les participants de l'expérience apparaissent donc appartenir à la même population que les répondants aux questionnaire.

Concernant les niveaux de sensibilité environnementale des participants de l'expérience, les mesures effectuées sur l'échelle NEP et sur la dimension « recyclage » de l'échelle 3R adaptée présentent des scores moyens supérieurs au niveau médian, respectivement de 4,02 (ET=0,62) pour la première et de 4,70 $(\mathrm{ET}=0,49)$ pour la seconde. La mesure effectuée sur la dimension 
Tableau 1

Dimensions de l'échelle 3R adaptée mises en évidence par l'analyse factorielle avec rotation Varimax.

Items de l'échelle 3R adaptée

23. Recycler le carton

21. Recycler les bouteilles en plastique

19. Recycler les journaux, magazines et dépliants publicitaires

20. Recycler les canettes, conserves et autres emballages alimentaires

24. Recycler les vêtements (dons aux associations par exemple)

14. Réutiliser le papier d'impression (verso des feuilles)

18. Recycler le verre

10. Acheter des fruits et légumes en vrac, non emballés

15. Réutiliser les bouteilles et/ou pots en verre

11. Acheter des produits qui peuvent être réutilisés, plutôt que des produits jetables

13. Essayer de réparer les objets avant d'en acheter de nouveaux

9. Choisir des produits dont l'emballage pourra être facilement réutilisés ou recyclés

25. Composter les déchets alimentaires

17. Réutiliser les contenants en plastique, comme les pots de glace par exemple

26. Composter les déchets végétaux (tontes de pelouse par exemple)

12 Consommer l'eau du robinet plutôt que de l'eau minérale en bouteille

7. Acheter des produits qui peuvent être réutilisés, plutôt que des produits jetables

8. Utiliser mon propre sac quand je fais les courses, plutôt que celui fourni par le magasin

16. Laver les chiffons plutôt que d'en acheter de nouveaux

22. Recycler le papier (documents administratifs, factures...)
Recyclage Réduction et

réutilisation

0,977

0,977

0,935

0,787

0,483

0,335

0,263

0,244

0,650

0,604

0,595

0,545

0,540

0,531

0,529

0,495

0,447

0,245

0,208

$-0,176$

L'italique indique un retrait de l'item.

« réduction et réutilisation » de l'échelle $3 \mathrm{R}$ adaptée présente pour sa part un niveau plus faible que les précédentes, bien que supérieur toutefois au niveau médian de l'échelle $(\mathrm{M}=3,50 ; \mathrm{ET}=0,71)$. Une différence significative entre la dimension « recyclage » et la dimension « réduction et réutilisation » est ainsi observée $[\mathrm{t}(1,68)=12,992 ; p=.0001)$.

Concernant la comparabilité des participants, nous nous sommes également intéressés aux participants des deux conditions de notre expérience. Les scores des individus sur ces différentes échelles ont été comparés après leur répartition dans les conditions de l'expérience. Aucune différence statistique n'est apparue (NEP : $[\mathrm{t}(1,37)=.713 ; p=.48 \mathrm{NS}] ; 3 \mathrm{R}$ dimension recyclage : $[\mathrm{t}(1,37)=.495 ; p=.62 \mathrm{NS}] ; 3 \mathrm{R}$ dimension réutilisation et réduction $:[\mathrm{t}(1,37)=.839 ; p=.41 \mathrm{NS}])$ présentant nos conditions comme comparables (Tableau 2).

\subsubsection{Test des hypothèses}

Parmi les 38 foyers initiaux ayant accepté de participer au projet, 4 foyers ont été retirés des analyses, du fait de problèmes techniques sur le système de puce électronique (3) et de l'impossibilité de mettre finalement en lien le numéro de puce et les réponses au questionnaire (1). Les deux conditions de l'expérience comptabilisaient finalement 17 foyers chacune. Les analyses préalables n'ont pas révélé de différences en ce qui concerne le nombre de personnes au foyer $(\mathrm{t}[1,33]=.385 ; p=.70 \mathrm{NS})$, le poids total de déchets généré sur l'année $2013(\mathrm{t}[1,33]=.670$; $p=.51 \mathrm{NS})$ ou le poids de déchets enregistrés sur la période pré-expérimentale $(\mathrm{t}[1,33]=.745$; $p=.46 \mathrm{NS})$.

Une première analyse a été réalisée au moyen d'une Anova factorielle à mesure répétée en considérant le poids moyen de déchets collectés lors des 3 périodes suivant la période pré-expérimentale (périodes expérimentale, post-expérimentale 1, post-expérimentale 2) comme variable intra-sujet 
Tableau 2

Moyennes et écarts-types des foyers selon leur acceptation ou refus du projet et selon leur condition d'appartenance.

\begin{tabular}{lcc}
\hline Échelle et dimension considérée & Moyennes & Écarts-types \\
\hline Échelle NEP & & 0,62 \\
$\quad$ Global & 4,02 & 0,62 \\
$\quad$ Foyers non volontaires (31) & 4,00 & 0,63 \\
$\quad$ Foyers volontaires (38) & 4,05 & 0,58 \\
$\quad$ Condition contrôle (20) & 3,98 & 0,68 \\
$\quad$ Condition feedback (18) & 4,12 & 0,49 \\
Échelle 3R adaptée dimension recyclage & & 0,64 \\
Global & 4,70 & 0,31 \\
$\quad$ Foyers non volontaires (31) & 4,63 & 0,38 \\
Foyers volontaires (38) & 4,77 & 0,22 \\
Condition contrôle (20) & 4,74 \\
$\quad$ Condition feedback (18) & 4,79 & 0,71 \\
Échelle 3R adaptée dimension réutilisation et réduction & & 0,70 \\
Global & 3,50 & 0,72 \\
$\quad$ Foyers non volontaires (31) & 3,48 \\
$\quad$ Foyers volontaires (38) & 3,51 & 0,63 \\
$\quad$ Condition Contrôle (20) & 3,61 & 0,82 \\
$\quad$ Condition Feedback (18) & 3,41 & \\
\hline
\end{tabular}

Tableau 3

Poids moyens hebdomadaire des déchets selon la période et la condition expérimentale et différence inter périodes (en $\mathrm{kg})$.

\begin{tabular}{llllll}
\hline Conditions & Périodes & Pré-expérimentale & Expérimentale & Post-expérimentale 1 & Post-expérimentale 2 \\
\hline Feedback & Moyenne & 9,10 & 7,24 & 7,69 & 7,55 \\
& ET & 5,69 & 4,35 & 4,09 & 4,38 \\
Contrôle & Moyenne & 7,81 & 8,03 & 7,79 & 7,91 \\
& ET & 4,26 & 3,62 & 3,60 & 4,32 \\
\hline
\end{tabular}

et la condition d'appartenance des participants comme variable inter-sujet. Les résultats n'ont pas révélé d'effet simple de la période $(\mathrm{F}[2,32]=.077 ; p=.93 \mathrm{NS})$, de la condition $(\mathrm{F}[1,33]=.046$; $p=.83 \mathrm{NS})$ ni d'interactions périodes X Condition $(\mathrm{F}[2,32]=.263 ; p=.77 \mathrm{NS})$. Les poids moyens par période sont présentés dans le Tableau 3.

Une seconde analyse, complémentaire a été réalisée à l'aide d'une série d'Anova univariées en considérant comme variable dépendante la différence de poids collectés dans les deux conditions entre deux périodes expérimentales, et comme variable indépendante la condition d'appartenance des participants. L'Anova réalisée sur la différence de poids entre la période expérimentale et la période pré-expérimentale présente un effet principal de la condition $\mathrm{F}(1,33)=3,985 ; p=.05$; $\phi=11)$. L'Anova réalisée sur la différence de poids entre la période pré-expérimentale et la période post-expérimentale 1 ne présente pas d'effet $\mathrm{F}(1,33)=.338 ; p=.56 \mathrm{NS})$. Enfin, l'Anova réalisée sur la différence de poids entre la période pré-expérimentale et la période post-expérimentale 2 ne présente pas non plus d'effet $(\mathrm{F}[1,33]=1,045 ; p=.31 \mathrm{NS})$. Le Tableau 4 synthétise les différences de poids inter-périodes.

\subsubsection{Analyses exploratoires}

Enfin, nous nous sommes intéressés aux liens existant entre les réponses des individus aux échelles de mesure de l'attitude et des comportements pro-environnementaux autorapportés 
Tableau 4

Différence inter-période de poids des déchets selon la condition expérimentale (en $\mathrm{kg}$ ).

\begin{tabular}{|c|c|c|c|}
\hline Conditions & $\begin{array}{l}\text { Différence } \\
\text { expérimentale } \\
\text { - pré-expérimentale }\end{array}$ & $\begin{array}{l}\text { Différence } \\
\text { post-expérimentale } 1 \\
\text { - pré-expérimentale }\end{array}$ & $\begin{array}{l}\text { Différence } \\
\text { post-expérimentale } 2 \\
\text { - pré-expérimentale }\end{array}$ \\
\hline \multicolumn{4}{|l|}{ Feedback } \\
\hline Moyenne & $-1,85$ & $-0,99$ & $-1,55$ \\
\hline ET & 3,35 & 5,71 & 5,86 \\
\hline \multicolumn{4}{|l|}{ Contrôle } \\
\hline Moyenne & 0,22 & $-0,04$ & $-0,09$ \\
\hline $\mathrm{ET}$ & 2,67 & 3,66 & 3,07 \\
\hline
\end{tabular}

Les données en gras révèlent une différence significative à $p=.05$.

recueillies lors du recrutement et le poids des déchets de l'année passé (2013) et le poids des 4 périodes de l'expérimentation. L'analyse corrélationnelle réalisée présente plusieurs liens significatifs. Ainsi, les 4 périodes de l'expérimentation présentent entre elles un lien significatif positif (pré-expérimentale et expérimentale : $\mathrm{r}(34)=.774 ; p=.0001)$ (pré-expérimentale et postexpérimentale $1 \mathrm{r}(34)=.556 ; p=.001)$ (pré-expérimentale et post-expérimentale $2: \mathrm{r}(34)=.499$; $p=.003$ ) (expérimentale et post-expérimentale $1 \mathrm{r}(34)=.616 ; p=.0001$ ) (expérimentale et postexpérimentale $2 \mathrm{r}(34)=.539 ; p=.001$ ) (post-expérimentale 1, post-expérimentale $2 \mathrm{r}(34)=.373$; $p=.03)$; un lien significatif est également observé entre le poids des déchets 2013 et les 4 périodes de l'expérimentation (pré-expérimentale : $\mathrm{r}(34)=.819 ; p=.0001)$, (expérimentale $\mathrm{r}(34)=.814$; $p=.0001$ ), (post-expérimentale $1 \mathrm{r}(34)=.641 ; p=.0001$ ), (post-expérimentale $2 \mathrm{r}(34)=.713$; $p=.0001)$. Enfin, une seule corrélation significative apparait entre les échelles de mesures administrées et les relevés effectifs de poids : le score de NEP présente un lien avec la mesure post-expérimentale $2 \mathrm{r}(34)=.353 ; p=.04)$. Le Tableau 5 synthétise ces résultats.

\subsection{Données qualitatives}

Les 17 foyers ayant participé au projet ont été contactés durant la période postexpérimentale 1 par téléphone afin de livrer leur ressenti sur l'étude et sur l'outil. Ces entretiens téléphoniques ont fait l'objet d'une analyse thématique du contenu.

\subsubsection{Ressenti concernant l'étude, l'outil mis à disposition et son utilisation}

L'étude a été favorablement perçue par les participants : aucun commentaire négatif n'a été émis et 7 répondants ont exprimé spontanément un commentaire positif sur l'étude. Un répondant a souhaité être informé des résultats et deux répondants ont exprimé le souhait de participer à une suite éventuelle du projet.

Les foyers ont bien perçu le rôle pédagogique de l'outil et leurs discours sur ce sujet apparaît spécifiquement dans les réponses de 7 d'entre eux aux différentes questions posées. Ces réponses mettent en évidence le fait que l'information permet de prendre conscience de la production de déchets («prise de conscience intéressante »; «c'est intéressant même si on avait déjà une prise de conscience importante, on recycle depuis longtemps »). Mais, ce sont surtout la répétition et l'historique qui permettent de prendre conscience de l'évolution des déchets, notamment en lien avec les activités du foyer («il y a des écarts en fonction de ce qu'on met dedans »; « la première pesée était élevée, on avait fait un vidage de placard... quand je jette la litière du chat, c'est lourd. .. »; «ça permet de se rendre compte si on est absent »; « on a vu qu' après un anniversaire, 
Tableau 5

Synthèse des corrélations observées.

\begin{tabular}{|c|c|c|c|c|c|c|c|c|c|c|c|c|}
\hline & M & ET & 1 & 2 & 3 & 4 & 5 & 6 & 7 & 8 & 9 & 10 \\
\hline 1. Âge & 38,08 & $\mathbf{9 , 8 2}$ & - & -.199 & -.226 & -.108 & -.317 & -.273 & -.251 & .304 & .329 & .269 \\
\hline 2. NombrePers foy & 3,65 & 1,178 & & - & $.514 *$ & $.522 *$ & $.555^{*}$ & $.434 *$ & .173 & -.329 & .029 & -.183 \\
\hline 3. Poids 2013 & 444,42 & 195,02 & & & - & $.819 *$ & $.814 *$ & $.641 *$ & $.713 *$ & -.226 & .077 & -.194 \\
\hline 4. Pré-exp & 8,45 & 4,99 & & & & - & $.774 *$ & $.556^{*}$ & $.499 *$ & -.186 & .019 & -.143 \\
\hline 5. Exp & 7,64 & 3,96 & & & & & - & $.616^{*}$ & $.539 *$ & -.297 & .130 & -.217 \\
\hline 6. Post-exp 1 & 7,94 & 5,08 & & & & & & - & $.373^{*}$ &,- 148 & -.195 & .106 \\
\hline 7. Post-exp 2 & 7,73 & 4,29 & & & & & & & - & $-.353^{*}$ & -.175 & -.173 \\
\hline 8. NEP & 4,06 & 649 & & & & & & & & - & .295 & .148 \\
\hline 9. Recyclage & 4,80 & .265 & & & & & & & & & - & -.087 \\
\hline 10. Réut. et Réduc. & 3,54 & .715 & & & & & & & & & & - \\
\hline
\end{tabular}

Les données en gras révèlent une différence significative à $p=.05$. Nombre pers Foy : nombre de personnes au foyer ; Pré-exp : période pré expérimentale ; Exp : période expérimentale ; Post exp 1 : période post-expérimentale 1 ; Post exp 2 : période post expérimentale 2 ; NEP : new environmental paradigm ; Reut et Réduc : réutilisation et recyclage. 
y'a des emballages cadeaux, des cartons, et c'est plus lourd »; « on a 2 ou 3 fois plus de poids après une fête, un anniversaire, un rangement »; « on voit que quand on fait la fête, c'est plus lourd »; «j'ai fait des travaux, donc j' ai jeté pas mal »; « on a mis la maison en vente, donc on a fait du tri et on a jeté »). D'autre part, le fait qu'il offre une alternative aux méthodes coercitives est très justement mentionné par l'un des répondants qui compare le feedback aux radars pédagogiques : « c'est comme les radars indicatifs... pas ceux qui donnent des amendes »; « on essaye de jouer le jeu, si on est dans le rouge, on essaye de revenir dans le vert ».

\subsubsection{L'évolution perçue des comportements}

Globalement, les répondants indiquent qu'ils n'ont pas modifié leurs pratiques (cette question obtient 11 réponses «non », 5 réponses plus modérées telles que «non mais », « je ne pense pas », « pas forcément » et 1 réponse « oui »). Le feedback a conforté de bonnes pratiques déjà en place : «ça a toujours été notre façon de faire »; « on essayait déjà de faire bien, ça a conforté nos habitudes »; et les changements ont pris place sans volonté explicite : «si on a baissé, c'est sans trop le vouloir, on n'y fait pas plus attention », « presque inconsciemment, l'évaluation régulière fait qu'on est un peu plus concerné... ».

Pour certains individus (3 répondants), l'outil semble avoir eu une influence plus marquée, les incitant à trier davantage (« on essaye de recycler un peu plus, on remplit un peu plus les sacs jaunes »; «avant on avait parfois la flemme de descendre au garage pour un carton »), ou à se lancer dans le compostage des déchet (« on trie plus les légumes, on a un conteneur au jardin qu'on a mis en place depuis »).

\subsubsection{Suggestions concernant des évolutions de l'outil}

Les suggestions proposées ont été relatives au mode de réception de l'information et à sa fréquence. En ce qui concerne le mode de réception de l'information, les retours ne permettent pas véritablement de trancher entre courriel et SMS (6 choix « SMS », 6 choix « mail », 3 choix SMS et/ou mail, 2 sans réponse).

En matière de fréquence de réception des informations, la fréquence hebdomadaire semble avoir été appréciée par les répondants, elle est citée 8 fois par les 11 sujets se prononçant) mais des périodicités bimensuelle ( 3 fois) ou mensuelle (4 fois) sont aussi suggérées, tandis qu'une fréquence trimestrielle n'est préférée qu'une fois.

Avec des périodes supérieures à une semaine, les fluctuations des poids des déchets sont lissées, ce qui en améliore la lisibilité.

\section{Discussion}

La présente étude avait pour objectif de tester l'effet d'un feedback hebdomadaire relatif au poids de déchets de foyers de l'agglomération rennaise sur l'évolution de ceux-ci. La littérature relative au recyclage des déchets et la pratique du tri présentant un effet positif et pérenne (lorsque cela est mesuré) de la procédure nous permettait de faire l'hypothèse d'une efficacité de l'outil. Quatre périodes d'expérimentation ont été définies sur près d'une année : une période pré-expérimentale de 7 semaines, une période expérimentale de 14 semaines et deux périodes post-expérimentales de 14 semaines chacune. Après s'être assuré que le poids de déchets collectés auprès des foyers des deux conditions ne différait pas sur la période pré-expérimentale, nous avons testé nos hypothèses à l'aide de deux analyses complémentaires. Dans un premier temps, une Anova factorielle à mesure répétée intrapériodes (expérimentale, post-expérimentale 1, post-expérimentale 2) a été réalisée de manière à mettre en évidence un effet d'interaction 
entre la condition d'appartenance des participants et la période de l'expérimentation et dans un second, une série d'Anova univariées afin de comparer l'ampleur de réduction inter-périodes (préexpérimentale - expérimentale, pré-expérimentale - post-expérimentale 1, pré-expérimentale post-expérimentale 2) entre les conditions. Les résultats de la première analyse ne révèlent pas d'effet d'interaction entre la condition d'appartenance des foyers et la période, ne confortant pas nos hypothèses 1a et $2 \mathrm{a}$. Les résultats de la seconde analyse présentent une différence significative entre les conditions Contrôle et Feedback sur la réduction de poids enregistrée entre la période pré-expérimentale et la période expérimentale, confortant notre hypothèse $1 \mathrm{~b}$. Ainsi, les foyers de la condition Feedback réduisent le poids de leurs déchets entre les deux périodes significativement plus que ceux de la condition contrôle. Par contre, cette seconde analyse ne conforte pas non plus notre hypothèse d'un effet durable de la procédure, qu'il s'agisse de la première période post-expérimentale ou de la seconde.

Les données collectées n'apparaissent cependant pas opposées à celles de la littérature. Après la diminution significative observée en condition Feedback entre la période pré-expérimentale et la période expérimentale, les poids enregistrés ne font pas montre d'un effet rebond, mais plutôt d'une stagnation. Pour illustrer notre propos, la proportion de réduction de $-20,44 \%$ calculée entre la période pré-expérimentale et la période expérimentale, s'établit à $-15,49 \%$ entre la période pré-expérimentale et la période post-expérimentale 1 , puis à $-17,03 \%$ entre la période pré-expérimentale et la période post-expérimentale 2. À titre de comparaison, les proportions d'évolution de poids de la condition Contrôle sont respectivement de $+2,82 \% ;-0,26 \%$ et $+1,28 \%$.

Deux éléments, vraisemblablement liés au protocole de recrutement ont pu concourir à la faiblesse statistique de nos résultats. D'une part, la taille de notre échantillon, comptabilisant 17 foyers par condition est particulièrement faible eu égard à la littérature. En comparaison, Schultz (1999) comptabilisait près de 120 participants par condition. D'autre part, la sensibilité environnementale dont témoignent les participants en amont de l'étude est relativement élevée et ne laisse, dès lors envisager, qu'une faible marge de progression, ce qui est conforté par le discours des répondants qui mentionne peu de changement. Le protocole de recrutement prévoyait un contact direct avec les foyers, la proposition d'un questionnaire préalable et un engagement à participer à une étude sans en connaître véritablement le contenu. Conçu pour s'assurer du caractère comparable et représentatif des participants (les échelles attitudinales et comportementales administrées ne présentent aucune différence entre les foyers des deux conditions, ni aucune différence entre les foyers ayant accepté ou refusé de participer au projet) et pouvoir n'imputer l'effet obtenu qu'à la seule réception régulière de l'information relative au poids des déchets résiduels, ce recrutement a pu, cependant limiter le nombre de participants et permettre la survenue d'un biais d'auto-sélection, constituant dès lors un faible échantillon d'individus particulièrement sensibles à l'environnement.

Afin de disposer d'un échantillon plus large et réellement représentatif, une future recherche devrait à la manière de l'instauration de la redevance incitative, informer l'ensemble des foyers sur la mise en place de l'action et transmettre directement l'information à une partie d'entre eux. Une enquête a posteriori permettrait alors d'évaluer la bonne réception de celle-ci et le niveau d'implication des foyers dans la démarche.

Malgré, les faiblesses d'échantillonnage pointées, un feedback sur le poids des ordures ménagères résiduelles, à l'instar d'un feedback sur les consommations d'énergie, sur la quantité ou la qualité de déchets recyclés produit un effet positif et laisse envisager que la procédure puisse constituer une alternative pertinente à la redevance incitative. En effet, la réduction des poids observés sur les 14 semaines de la période expérimentale de notre recherche (-20\%) est compa- 
rable à celle observée dans les recherches mesurant l'effet de la redevance incitative (Callan \& Thomas, 2006 ; Dahlén et al., 2009 ; Allers \& Hoeben, 2010). Du point de vue des foyers, l'approche pédagogique du feedback, non coercitive est bien accueillie. La possibilité de suivre l'évolution des poids semaines après semaines est pointée comme un élément déterminant de la prise de conscience et peut donc expliquer l'effet obtenu. L'historique des poids apporte en effet du contrôle aux foyers, qui dès lors peuvent passer en revue leurs comportements passés, déterminer les facteurs d'augmentation de poids et agir en conséquence. Les retours des foyers nous indiquent également que la réduction peut se faire sans nécessiter de changement profond des habitudes, simplement en faisant un peu plus attention. Ce qui pourrait motiver les plus gros producteurs de déchets à s'impliquer dans la démarche. Du point de vue des collectivités, l'approche par feedback est peu susceptible de susciter des incivilités de type dépôts sauvages, erreurs volontaires de tri, dépôt dans les communes voisines. En l'absence de sanction, ces pratiques ne présentent en effet aucun intérêt. Enfin, dès lors que le système de mesure ne donne pas lieu à une facturation, son niveau de précision est moins critique, ce qui pourrait permettre aux collectivités de faire le choix d'un système moins coûteux.

Notre protocole expérimental prévoyait l'administration préalable d'échelles de mesure de l'attitude pro-environnementale générale (NEP), d'une part, et de comportements autorapportés de gestion des déchets, d'autre part (3R). L'inclusion de ces échelles visait, d'une part, à faciliter le recrutement des participants en les engageant à l'aide d'une requête préalable peu coûteuse (Freedman \& Fraser, 1966), d'autre part, à mesurer la sensibilité environnementale des individus, recenser leur comportements de gestion des déchets et explorer les relations entres ces indicateurs et le poids effectif de déchets collectées de sorte à enrichir la littérature relative à la réduction des déchets. Globalement, comme indiqué précédemment, les foyers présentent une sensibilité environnementale relativement élevée. Les scores enregistrés sont en effet tous supérieurs au niveau médian des échelles utilisées qu'il s'agisse de l'attitude ou des comportement autorapportés de gestion des déchets. Concernant les comportements autorapportés plus précisément, nous observons une supériorité statistique des scores moyens de la dimension « Recyclage » par rapport à la dimension « réduction et réutilisation ». Conformément aux recherches de Barr et al. (2001) et Tonglet et al. (2004), ce résultat confirme le fait que les efforts des praticiens doivent désormais se focaliser sur ce second lot de comportements. L'analyse corrélationnelle réalisée ne présente pour sa part que peu de liens significatifs entre les scores obtenus aux échelles et les données effectives de poids recueillies. En effet, seule une corrélation entre le score d'attitude NEP et la mesure de poids réalisées sur la seconde période post-expérimentale rencontre la significativité statistique. S'agissant des comportements autorapportés, pourtant utilisés comme des indicateurs dans de nombreuses expérimentations, ils ne présentent ici aucun lien statistique avec l'attitude, d'une part, et avec les données de poids effectifs, d'autre part. La faiblesse de notre effectif pourrait expliquer cette absence de lien ; une forme de désirabilité sociale des réponses également. Félonneau \& Becker (2008) indiquaient à cet effet, qu'en situation d'autovalorisation, les individus présentaient des niveaux d'attitude et de comportements pro-environnementaux plus élevés qu'en situation standard. Le souhait d'apparaître sous un angle favorable auprès de l'expérimentatrice a pu conduire les individus à majorer leur implication environnementale. Une recherche mettant en relation attitudes, comportements rapportés et données effectives de poids de déchets d'ordures ménagères résiduelles auprès d'un échantillon plus conséquent de participants serait pertinente, afin d'établir de manière solide le niveau de validité des indicateurs comportementaux utilisés.

Cette recherche en appelle d'autres également, en ce qui concerne les modalités de feedback. S'agissant d'une première expérimentation, il est nécessaire de répliquer l'effet obtenu et travailler son maintien sur le long terme. À partir du retour des participants, le mode de réception par 
courriel plus SMS semble pertinent puisqu'il permet de combiner l'immédiateté de l'information et la conservation de celle-ci dans un historique. La fréquence d'envoi pourrait pour sa part faire l'objet d'une recherche comparant différentes modalités. S'agissant du contenu informatif transmis, outre le poids des déchets et l'historique, des investigations sont nécessaires afin d'évaluer l'effet additif du couplage de l'information à un objectif de réduction tel que cela est fait dans certaines recherches sur la diminution des consommations d'énergie (Becker, 1978). Un couplage avec de l'information pratique de type « astuces de réduction » serait également pertinent. En effet, si le feedback permet de prendre conscience des poids générés selon le comportement, des astuces de réduction pourraient être directement testées par les foyers sur une période donnée et démontrer leur efficacité, ce qui, le cas échéant, favoriserait leur adoption. Enfin, un couplage à de l'information sociale relative au poids moyen de déchets produits par des foyers comparables pourrait également faire l'objet d'investigations. À cet effet, les résultats de Schultz (1999) observant un effet significatif en phase expérimentale et post-expérimentale d'un tel feedback offre des perspectives prometteuses.

\section{Déclaration de liens d'intérêts}

Les auteurs déclarent ne pas avoir de liens d'intérêts.

\section{Remerciements}

Cette recherche a été réalisée à l'aide d'un financement de la Maison des sciences humaines de Bretagne (MSHB) que nous remercions.

\section{Annexe A. Matériel complémentaire}

Le matériel complémentaire accompagnant la version en ligne de cet article est disponible sur http://www.sciencedirect.com et http://dx.doi.org/10,1016/j.rhum.2007,06.020.

\section{Références}

ADEME. (2015). Déchets : chiffres clés. http://www.ademe.fr/dechets-chiffres-cles. Consulté le 19/05/2016.

Ajzen, I. (1991). The theory of planned behavior. Organizational Behavior and Human Decision Processes, 50(2), 179-211. http://dx.doi.org/10.1016/0749-5978(91)90020-T

Allers, M. A., \& Hoeben, C. (2010). Effects of unit-based garbage pricing: A differences-in-differences approach. Environmental and Resource Economics, 45(3), 405-428. http://dx.doi.org/10.1007/s10640-009-9320-6

Bamberg, S., \& Moser, G. (2007). Twenty years after Hines, Hungerford, and Tomera: a new meta-analysis of psychosocial determinants of pro-environmental behaviour. Journal of Environmental Psychology, 27(1), 14-25. http://dx.doi. org/10.1016/j.jenvp.2006.12.002

Barr, S., Gilg, A. W., \& Ford, N. J. (2001). Differences between household waste reduction, reuse and recycling behaviour: A study of reported behaviours, intentions and explanatory variables. Waste Management, 4(2), 69-82.

Becker, L. J. (1978). Joint effect of feedback and goal setting on performance: A field study of residential energy conservation. Journal of Applied Psychology, 63(4), 428-433. http://dx.doi.org/10.1037//0021-9010.63.4.428

Botetzagias, I., Dima, A. F., \& Malesios, C. (2015). Extending the theory of planned behavior in the context of recycling: The role of moral norms and of demographic predictors. Resources, Conservation and Recycling, 95, 58-67. http://dx. doi.org/10.1016/j.resconrec.2014.12.004

Burn, S. M., \& Oskamp, S. (2013). Increasing community recycling with persuasive communication and public commitment. Journal of Chemical Information and Modeling, 53(9), 1689-1699. http://dx.doi.org/10. 1017/CBO9781107415324.004 
Callan, S. J., \& Thomas, J. M. (2006). Analyzing demand for disposal and recycling services: A systems approach. Eastern Economic Journal, 32, 221-240.

Dahlén, L., Åberg, H., Lagerkvist, A., \& Berg, P. E. O. (2009). Inconsistent pathways of household waste. Waste Management, 29(6), 1798-1806. http://dx.doi.org/10.1016/j.wasman.2008.12.004

Darby, S. (2006). The effectiveness of feedback on energy consumption. Environmental Change Institute University of Oxford, 22(April), 1-21. http://dx.doi.org/10.4236/ojee.2013.21002

Davies, J., Foxall, G. R., \& Pallister, J. (2002). Beyond the intention-behaviour mythology. An integrated model of recycling. Marketing Theory, 2(1), 29-113. http://dx.doi.org/10.1177/1470593102002001645

Deci, E. L., \& Ryan, R. M. (1988). Intrinsic motivation and self-determination in human behavior. Contemporary Sociology, 17 http://dx.doi.org/10.2307/2070638

De Young, R. (1990). Recycling as appropriate behavior: A review of survey data from selected recycling education programs in Michigan. Resource Conservation and Recycling, 3, 253-267.

Dunlap, R. E., Liere, K. D., Van Mertig, A. G., \& Jones, R. E. (2000). Measuring endorsement of the new ecological paradigm: A revised NEP scale. Journal of Social Issues, 56(3), 425-442. http://dx.doi.org/10.1111/0022-4537.00176

Dupré, M. (2009). De l'engagement comportemental à la participation, exploration de stratégies de communication appliquées au tri et à la prévention des déchets ménagers (Thèse de doctorat [non publiée]). Rennes: Université de Haute Bretagne.

Dupré, M., Dangeard, I., \& Meineri, S. (2014). Revue de littérature sur les techniques d'influence et de communication appliquées à la gestion des déchets. Pratiques Psychologiques, 20(4), 211-230. http://dx.doi.org/10. 1016/j.prps.2014.09.003

Dupré, M., Meineri, S., \& Guéguen, N. (2014). Communication engageante appliquée au tri sélectif : comparaison entre les techniques du pied-dans-la-porte et du double-pied-dans-la-porte. Les Cahiers Internationaux de Psychologie Sociale, 102(2), 259. http://dx.doi.org/10.3917/cips.102.0259

Félonneau, M. L., \& Becker, M. (2008). Pro-environmental attitudes and behavior: Revealing perceived social desirability. Revue Internationale de Psychologie Sociale, 21(4), 25-53.

Freedman, J. L., \& Fraser, S. C. (1966). Compliance without pressure: the foot-in-the-door technique. Journal of Personality and Social Psychology, 4(2), 195-202 [http://doi.org/10.1037/h0023552].

Fullerton, D., \& Kinnaman, T. C. (1996). Household responses to pricing garbage by the bag. American Economic Review, 86(4), 971-984 [http://doi.org/10.1257/jep.6.3.79].

Fuqua, R., \& Deleon, I. (1995). The effects of public commitment and group feedback on curbside recycling. Environment and Behavior, 27(2), 233-250. http://dx.doi.org/10.1177/0013916595272007

Gardner, G., \& Stern, P. (1996). Environmental problems and human behavior. Boston: Allyn \& Bacon.

Gatier, A. (2016). La tarification incitative de la gestion des ordures ménagères - Quels impacts sur les quantités collectées ? Collection «Études et documents » du Service de l'économie, de l'évaluation et de l'intégration du développement durable (SEEIDD) du Commissariat général au développement durable (CGDD), 140, $1-60$.

Geller, E. S. (1992). It takes more than information to save energy. American Psychologist, 1(June), 814-815. http://dx.doi.org/10.1017/CBO9781107415324.004

Geller, E. S., Chaffee, J. L., \& Ingram, R. E. (1975). Promoting paper-recycling on a university campus. Journal of Environmental Systems, 5(1), 39-57. http://dx.doi.org/10.2190/E2LM-JNTV-NBJ6-ETJF

Goldenhar, L. M., \& Connell, C. M. (1991-1992). Effects of education and feedback interventions on recycling knowledge, attitudes, beliefs, and behaviors. Journal of Environmental Systems, 21, 321-333.

Guéguen, N., Meineri, S., Martin, A., \& Grandjean, I. (2010). The combined effect of the foot-in-the-door technique and the "but you are free" technique: An evaluation on the selective sorting of household wastes. Ecopsychology, 2(4), 231-237. http://dx.doi.org/10.1089/eco.2009.0051

Hamad, C. D., Bettinger, R., Cooper, D., \& Semb, G. (1980). Using behavioral procedures to establish an elementary school paper recycling program. Journal of Environmental Systems, 10, 149-156.

Hong, S. (1999). The effects of unit pricing system upon household solid waste management: The Korean experience. Journal of Environmental Management, 57(1), 1-10. http://dx.doi.org/10.1006/jema.1999.0286

Hopper, J. R., \& Nielsen, J. M. (1991). Recycling as altruistic behavior: Normative and behavioral strategies to expand participation in a community-recycling program. Environment and Behavior, 23(2), 195-220. http://dx.doi.org/10.1177/0013916591232004

Humphrey, C. R., Bord, R. J., Hammond, M. M., \& Mann, S. H. (1977). Attitudes and conditions for cooperation in a paper-recycling program. Environment and Behavior, 9(1), 107-124. http://dx.doi.org/10.1177/001391657791007

Iyer, E. S., \& Kashyap, R. K. (2007). Consumer recycling: role of incentives, information, and social class. Journal of Consumer Behaviour, 6(1), 32-47, http://doi.org/10.1002/cb.206. 
Jacobs, H. E., \& Bailey, J. S. (1982). Evaluating participation in a residential recycling program. Journal of Environmental Systems., http://dx.doi.org/10.2190/KWWT-HCX3-F1WG-WCEP

Jacobs, H. E., Bailey, J. S., \& Crews, J. I. (1984). Development and analysis of a community-based resource recovery program. Journal of Applied Behavior Analysis, 17, 127-145.

Joule, R. V., \& Beauvois, J. L. (1998). La Soumission Librement Consentie. Paris: Presses Universitaires de France.

Katzev, R., Cooper, L., \& Fisher, P. (1981). The effect of feedback and social reinforcement on residential electricity consumption. Journal of Environmental Systems, 10(3), 215-227. http://dx.doi.org/10.2190/M8W4JUYV-NB03-CUFL

Katzev, R. D., \& Pardini, A. U. (1988). The comparative effectiveness of reward and commitment approaches in motivating community recycling. Journal of Environmental Systems, 17(2), 93-113.

Katzev, R., \& Mishima, H. R. (1992). The use of posted feedback to promote recycling. Psychological Reports, 71, 259-264. http://dx.doi.org/10.2466/pr0.1992.71.1.259

Kiesler, C. (1971). The psychology of commitment. Academic Press.

Lehman, P. K., \& Geller, E. S. (2005). Behavior analysis and environmental protection: Accomplishments and potential for more. Behavior and Social Issues, 13(1) (Spring/Summer 2004). Retrieved from http://ojphi.org/ojs/index.php/bsi/article/view/33.

Linderhof, V., Kooreman, P., Allers, M., \& Wiersma, D. (2001). Weight-based pricing in the collection of household waste: The Oostzaan case. Resource and Energy Economics, 23(4), 359-371. http://dx.doi.org/10.1016/ S0928-7655(01)00044-6

Luyben, P. D., \& Bailey, J. S. (1979). Newspaper recycling: The effects of rewards and proximity of containers. Environment and Behavior, 11(4), 539-557. http://dx.doi.org/10.1177/0013916579114006

Needleman, L. D., \& Geller, E. S. (1992). Comparing interventions to motivate work-site collection of home-generated recyclables. Journal of Community Psychology, 20, 775-785.

Nickerson, R. S. (2003). In N. L. Mahwah (Ed.), Psychology and environmental change. Lawrence Erlbaum Associates, Publishers.

Nigbur, D., Lyons, E., Uzzell, D., \& Muckle, R. (2005). Increasing recycling through community action. Guildford: Department of Psychology, University of Surrey.

Oskamp, S. (1995). Resource conservation and recycling: Behavior and policy. Journal of Social Issues, 51(4), 157-177. http://dx.doi.org/10.1111/j.1540-4560.1995.tb01353.x

Pallak, M. S., \& Cummings, W. (1976). Commitment and voluntary energy conservation. Personality and Social Psychology Bulletin, 2(1), 27-30. http://dx.doi.org/10.1177/014616727600200105

Pardini, A. U., \& Katzev, R. D. (1983). The effect of strength of commitment on newspaper recycling. Journal of Environmental Systems, 13(3), 245-254. http://dx.doi.org/10.2190/6PN9-MXFP-3BFF-CHHB

Perrin, D., \& Barton, J. (2001). Issues associated with transforming household attitudes and opinions into materials recovery: A review of two kerbside recycling schemes. Resources, Conservation and Recycling, http://dx.doi.org/10.1016/S0921-3449(01)00075-1

Porter, B. E., Leeming, F. C., \& Dwyer, W. O. (1995). Solid waste recovery: A review of behavioral programs to increase recycling. Environment \& Behavior, 27(2), 122-152 [http://doi.org/0803973233].

Robertson, S., \& Walkington, H. (2009). Recycling and waste minimisation behaviours of the transient student population in Oxford: Results of an on-line survey. Local Environment, 14(4), $285-296$. http://dx.doi.org/10.1080/13549830902812982

Schultz, P. W. (1999). Changing behavior with normative feedback interventions: A field experiment on curbside recycling. Basic and Applied Social Psychology, 21(1), 25-36. http://dx.doi.org/10.1207/ s15324834basp2101_3

Schultz, P. W., Oskamp, S., \& Mainieri, T. (1995). Who recycles and when? A review of personal and situational factors. Journal of Environmental Psychology, 15(2), 105-121. http://dx.doi.org/10.1016/0272-4944(95)90019-5

Skinner, B. F. (1953). Science and human behavior. Journal of the experimental analysis of behavior, 80 http://dx.doi.org/10.1901/jeab.2003.80-345

Thorndike, E. L. (1998). Animal intelligence: An experimental study of the associate processes in animals (originally published 1898). American Psychologist, 53(10), 1125-1127.

Timlett, R. E., \& Williams, I. D. (2008). Public participation and recycling performance in England: A comparison of tools for behaviour change. Resources, Conservation and Recycling, 52(4), 622-634. http://dx.doi.org/10.1016/j.resconrec.2007.08.003

Tonglet, M., Phillips, P. S., \& Read, A. D. (2004). Using the theory of planned behaviour to investigate the determinants of recycling behaviour: A case study from Brixworth, UK. Resources. Conservation and Recycling, 41(3), $191-214$. http://dx.doi.org/10.1016/j.resconrec.2003.11.001 
Wang, T. H., \& Katzev, R. D. (1990). Group commitment and resource conservation: Two field experiments on promoting recycling. Journal of Applied Social Psychology, 20(4), 265-275. http://dx.doi.org/10.1111/j. 1559-1816.1990.tb00411.x

Webb, T. L., \& Sheeran, P. (2006). Does changing behavioral intentions engender behavior change? A meta-analysis of the experimental evidence. Psychological Bulletin, 132(2), 249-268. http://dx.doi.org/10.1037/0033-2909.132.2.249

Werner, C. M., Turner, J., Shipman, K., Shawn Twitchell, F., Dickson, B. R., Bruschke, G. V., \& von Bismarck, W. B. (1995). Commitment, behavior, and attitude change: An analysis of voluntary recycling. Journal of Environmental Psychology, 15(3), 197-208. http://dx.doi.org/10.1016/0272-4944(95)90003-9

Winett, R. A., Neale, M. S., \& Grier, H. C. (1979). Effects of self-monitoring and feedback on residential electricity consumption. Journal of Applied Behavior Analysis, 12(2), 173-184. http://dx.doi.org/10.1901/jaba.1979.12-173

Witmer, J. F., \& Geller, E. S. (1976). Facilitating paper recycling: effects of prompts, raffles, and contests. Journal of Applied Behavior Analysis, 9(3), 315-322. http://dx.doi.org/10.1901/jaba.1976.9-315

Woodard, R., Firoozmand, F., \& Harder, M. K. (2006). The use of cash vouchers to incentive householders to recycle. In Cristina, \& Zhang (Eds.), Waste conferences series (pp. 477-486).

Zbinden, A., Souchet, L., Girandola, F., \& Bourg, G. (2011). Communication engageante et représentations sociales : une application en faveur de la protection de l'environnement et du recyclage. Pratiques Psychologiques, 17, $285-299$. http://dx.doi.org/10.1016/j.prps.2010.10.002 